Ethiopian Journal of Environmental Studies \& Management 9(6): 691 - 699, 2016.

ISSN:1998-0507

Submitted: April 08, 2016

doi: http://dx.doi.org/10.4314/ejesm.v9i6.3

\title{
ASSESSMENT OF PHYSICO-CHEMICAL PARAMETERS OF RIVER KADUNA AND COLLEGE OF AGRICULTURE AND ANIMAL SCIENCE DAM, (CAAS) KADUNA, NIGERIA
}

\author{
*OJUTIKU, R.O., HABIBU, S., KOLO, R.J. AND OYERO, J.O. \\ Department of Water Resources, Aquaculture and Fisheries Technology, Federal \\ University of Technology, Minna, Niger State, Nigeria
}

\begin{abstract}
River Kaduna is one of the major sources of water to the inhabitants of the communities around the river and also to fish farmers while College of Agriculture and Animal Science Dam (CAAS) is used for fish farming and also for domestic purpose. The study was carried out to assess Physicochemical Parameters of River Kaduna and College of Agriculture and Animal Science Dam, Kaduna. The result of seasonal variation of physicochemical parameters between River Kaduna and College of Agriculture and Animal Science Dam, revealed that the atmospheric temperature (AT) ranged from 32.8 to $33.6^{\circ} \mathrm{C}, \mathrm{pH}$ at 7.1 , conductivity ranged from 83.7 to $139.7 \mu^{\mathrm{s}} / \mathrm{cm}$, dissolved oxygen ranged from 9.2 to 9.4 $\mathrm{mg} / \mathrm{l}$, biological oxygen demand ranged from 5.2 to $5.9 \mathrm{mg} / \mathrm{l}$, water temperature (WT) at $29.2^{\circ} \mathrm{C}$, total hardness ranged from 37.4 to $58.0 \mathrm{mg} / \mathrm{l}$, alkalinity ranged from 18.8 ton 29.7 $\mathrm{mg} / \mathrm{l}$, chloride ranged from 14.8 to $15.9 \mathrm{mg} / \mathrm{l}$, nitrate and phosphorus ranged from 0.1 to $0.2 \mathrm{mg} / \mathrm{l}$ while transparency ranged from 21.4 to $22.3 \mathrm{~cm}$ respectively. The seasonal variation result revealed that both at the Dam and river, $A T, p H, D O, W T$, Chloride, Nitrate, Phosphorus and Transparency values were not significantly different while conductivity, BOD, TH, Alkalinity and Ammonia were significantly different. However, they were significantly difference from one month to another (wet and dry season months respectively).
\end{abstract}

Key Words: Physico-chemical parameters, River Kaduna, CAAS Dam

\section{Introduction}

Pollution of a river first affects its chemical quality and then systematically destroys the community disrupting the delicate food web. Diverse uses of the rivers are seriously impaired due to pollution and even the polluters like industry suffer due to increased pollution of the rivers. River pollution has several dimensions and effective monitoring and control of river pollution requires the expertise from various disciplines (Trivedy et al., 1990) Pollution of river is a global problem. In India it is reported that about $70 \%$ of the available water is polluted.

Physicochemical parameters include; temperature, transparency, dissolved oxygen, hardness, alkalinity, $\mathrm{pH}$, conductivity, biochemical oxygen 
demand, etc. Water is a medium which permit the existence of both living and non-living organisms. Seasonal variations in physiochemical parameters affect the living organisms such as phytoplankton and zooplankton living in the water body in terms of their distributions and abundance. The global environment is changing continuously due to unfavourable alteration of surroundings, wholly as a byproduct of man's actions, through direct or indirect effects of changes in energy pattern, radiation levels, chemical and physical constitution of organisms. These changes may affect man directly or through his supplies of water and of agricultural and other biological products. Chemicals are a major source of water contamination (Kataria, 2004) that introduced during water movement through geological materials, manufactured chemicals may cause problems.

Fertilizers and pesticides are major contributors to water pollution, Nitrates from fertilizers are a common chemical pollutant of water. Heavy metals, sulphates, nitrates, chlorides, phosphates, carbonates, ammonia, pesticides, phenols, soaps, detergents are the common chemical pollutants.

Physicochemical parameter has been used to access water bodies' health. In Africa, Nigeria in particular, there are existing laws governing the water ways, but yet these laws are not properly enforced, thus, leading to decrease in the quality of water bodies as a result of various anthropogenic activities within the riparian and other communities (Edegbene and Arimoro, 2012). Changes in the physical and chemical characteristics of Ibiekuma stream after impoundment were investigated by Edokpayi and Osimen (2002) and found that, fluctuations in the physical and chemical hydrology of the stream appear to be influenced by the changes in the fluvial dynamics of the stream due to impoundment. Asonye et al. (2007) reported that turbidity and conductivity were far above the acceptable limits recommended by WHO in some Nigeria streams and water ways.

Pollution indicators in River Kaduna, Kaduna state, Nigeria was determined by Mahre et al. (2007) and found that some of the parameters were above the recommended permissible limits set by Federal Environmental Protection Agency (FEPA), 2000, Nigeria, World Health Organization (WHO), 2004/European Union (EU). They suggested a protective measures to be taken so that the high concentration of the different physic-chemical variables not to have deleterious effect on aquatic ecosystem and the health of the rural dwellers who use the water for domestic purpose without treatment.

Edegbene et al. (2012) reported that rivers are fast deteriorating which could be attributed to human activities and other anthropogenic activities. Water quality assessment generally involves analysis of physicochemical, biological and microbiological parameters and reflects on abiotic and biotic status of the ecosystem (Rajagopal et al., 2011). This study assessed the quality of the CAAS dam and River Kaduna and its implication to aquaculture. 


\section{Materials and Method}

\section{Study Area}

This was carried out at College of Agriculture and Animal Science (CASS) dam, Mando Road Kaduna and River Kaduna within the city of Kaduna metropolis which is located in the northern part of the country bounded by latitude $\mathrm{N} 10^{\circ} 35^{\prime} .531^{\prime \prime}$, longitude $007^{\circ}$ $25^{\prime} .414^{\prime \prime}$ and latitude N $10^{\circ} 28^{\prime} .927^{\prime \prime}$, longitude $\quad 007^{\circ} \quad 23^{\prime} .616^{\prime \prime}$.

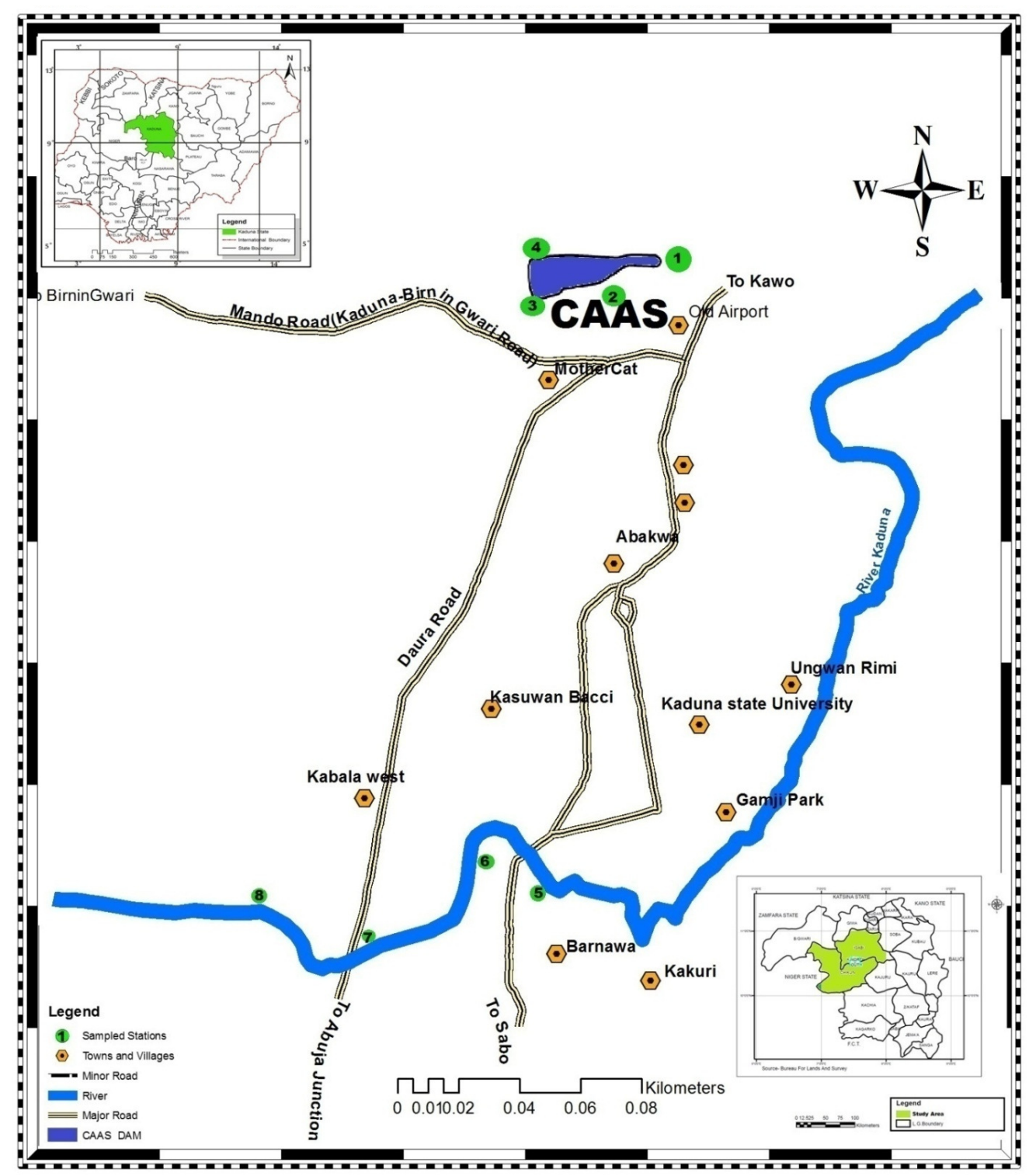

Figure 1: Map of Kaduna metropolis showing CAAS Dam and River Kaduna with the sampling stations 


\section{Water Sampling}

Water samples were collected once in a month for a period of twelve months from four sampling stations each from the two water bodies as shown on table 1 between the hours of 8:00 am to 1:00 pm as described by Burn and David (1999).
Samples were transferred to the Laboratory of the Water Resources, Aquaculture and Fisheries Technology Department, Federal University of Technology, Minna for the analysis of physico-chemical parameters.

Sampling Stations Co-ordinate Point

\begin{tabular}{|c|c|c|c|c|}
\hline \multicolumn{5}{|c|}{ UTM Co-ordinate } \\
\hline & & CAAS DAM & & \\
\hline Stations & $\mathrm{X}$-coordinate & Y-coordinate & Latitude $(\mathrm{N})$ & Longitude (E) \\
\hline 1 & 0329529 & 1171217 & 1035531 & 00725414 \\
\hline 2 & 0327618 & 11712171 & 1035559 & 00715463 \\
\hline 3 & 0327826 & 1171269 & 1035560 & 00725577 \\
\hline 4 & 0327956 & 1171227 & 1035537 & 00725649 \\
\hline \multicolumn{5}{|c|}{ RIVER KADUNA } \\
\hline 1 & 0327755 & 1171162 & 1035501 & 00725538 \\
\hline 2 & 0327572 & 1171159 & 1035499 & 00725438 \\
\hline 3 & 0327343 & 1171413 & 1035459 & 0725647 \\
\hline 4 & 0327657 & 11619515 & 1035559 & 0727589 \\
\hline
\end{tabular}

Physico-chemical parameters analyze include; Air temperature (AT), Water Temperature (WT), Transparency (Trans), Total Hardness (TH), Total Alkalinity (TA), Conductivity (Condu), Dissolved oxygen (DO), Biochemical oxygen demand (BOD), and Hydrogen ion concentration $(\mathrm{pH})$, Ammonia, Nitrates, phosphate and chloride. Meanwhile, AT, WT and Transparency were analyzed in the field. These analyses were done using the method of APHA, 1995.

\section{Method of Analysis}

The data collected were subjected to analysis of variance (ANOVA) and principal component analysis (PCA) to assess the effects and correlations of sample by stations and monthly variation of the physico-chemical parameters.

\section{Results and Discussion}

The results of this study as presented in the tables 2, 3, 4, 5, 6 and figure $2,3,4,5$. Water temperature of CAAS dam was between $28.6^{\circ} \mathrm{C}-29.6^{\circ} \mathrm{C}$ across the stations while dissolved oxygen was within the range of $8.1 \mathrm{mg} / \mathrm{L}-10.1 \mathrm{mg} / \mathrm{l}$, nitrate and ammonia range were 0.1 and $0.1-0.9 \mathrm{mg} / \mathrm{L}$ respectively. Table 2 shows the monthly variations in the parameters measured during the study period and significant difference $(\mathrm{P}<0.05)$ were observed in some of the parameters. Dissolved oxygen ranged between $6.0-10.8 \mathrm{mg} / \mathrm{L}$. Table 4 showed that water temperature ranged between $28.2-30.3^{0} \mathrm{C}$, dissolved oxygen between $9.1-10.0 \mathrm{mg} / \mathrm{L}$ and ammonia recorded was $0.2 \mathrm{mg} / \mathrm{L}$ across the stations in River Kaduna while monthly variations was also observed in all the parameters measured as shown on Table 5. 
Figure 2 and 3 shows the correlation of physico-chemical parameters in CAAS dam and River Kaduna respectively. It shows that conductivity, $\mathrm{pH}$, Transparency, alkalinity, total hardness and air temperature were positively correlated while alkalinity, water temperature, conductivity, air temperature, total hardness and $\mathrm{pH}$ were negatively correlated as shown in figure 2 for CAAS dam.

Figure 3 revealed that total hardness, $\mathrm{pH}$, conductivity, transparency were positively correlated while BOD, phosphate, transparency and water temperature were negatively correlated. It was revealed that physico-chemical parameters of both CAAS Dam and River Kaduna shows that some parameters were within desirable limits for fish culture and some were above desirable limits for fish culture.
The results of physico-chemical parameters on the dam and river during the study period revealed that atmospheric temperature ranged from $32.8-33.6^{\circ} \mathrm{C}$ which is in line with Madu et al. (2003); $\mathrm{pH}$ value agreed with the earlier report Ovie (2011); Dissolved oxygen ranged from $9.2-9.4 \mathrm{mg} / \mathrm{l}$ though a minimum constant of $5 \mathrm{mg} / \mathrm{l}$ is satisfactory (Wade, 1992). The DO, AT, $\mathrm{pH}$, chloride, Nitrate, Phosphorus, Ammonia and Transparency were not significantly different $(\mathrm{P}>0.05)$ while Conductivity, BOD, TH and Alkalinity were significantly different $(\mathrm{P}<0.05)$. However, there was fluctuation across the months and period of sampling of these parameters which is supported by earlier report of Ojutiku and Kolo, (2008); Ojutiku and Kolo (2011).

Table 2: Stations Variation of Physicochemical Parameters for CAAS Dam

\begin{tabular}{llllll}
\hline \multicolumn{5}{c}{ Stations } \\
\hline Parameters & 1 & 2 & 3 & 4 & \pm SE \\
\hline $\mathrm{AT}\left({ }^{0} \mathrm{C}\right)$ & $32.8^{\mathrm{a}}$ & $34.0^{\mathrm{a}}$ & $33.6^{\mathrm{a}}$ & $34.1^{\mathrm{a}}$ & 0.5 \\
$\mathrm{WT}\left({ }^{0} \mathrm{C}\right)$ & $29.5^{\mathrm{a}}$ & $28.6^{\mathrm{a}}$ & $29.2^{\mathrm{a}}$ & $29.6^{\mathrm{a}}$ & 0.4 \\
$\mathrm{P}^{\mathrm{H}}$ & $7.2^{\mathrm{a}}$ & $7.1^{\mathrm{a}}$ & $7.1^{\mathrm{a}}$ & $7.1^{\mathrm{a}}$ & 0.1 \\
$\mathrm{Cond} .(\mu \mathrm{s} / \mathrm{cm})$ & $108.5^{\mathrm{a}}$ & $74.7^{\mathrm{a}}$ & $75.5^{\mathrm{a}}$ & $76.7^{\mathrm{a}}$ & 9.9 \\
$\mathrm{DO}(\mathrm{mg} / \mathrm{l})$ & $9.7^{\mathrm{a}}$ & $10.1^{\mathrm{a}}$ & $9.1^{\mathrm{a}}$ & $8.1^{\mathrm{a}}$ & 9.9 \\
$\mathrm{BOD}(\mathrm{mg} / \mathrm{l})$ & $5.6^{\mathrm{a}}$ & $5.2^{\mathrm{a}}$ & $5.4^{\mathrm{a}}$ & $4.8^{\mathrm{a}}$ & 0.4 \\
$\mathrm{TH}(\mathrm{mg} / \mathrm{l})$ & $31.2^{\mathrm{a}}$ & $38.3^{\mathrm{a}}$ & $39.0^{\mathrm{a}}$ & $41.0^{\mathrm{a}}$ & 3 \\
$\mathrm{ALK}(\mathrm{mg} / \mathrm{l})$ & $18.8^{\mathrm{a}}$ & $20.0^{\mathrm{a}}$ & $18.2^{\mathrm{a}}$ & $18.0^{\mathrm{a}}$ & 1.8 \\
$\mathrm{Cl}(\mathrm{mg} / \mathrm{l})$ & $15.5^{\mathrm{a}}$ & $12.9^{\mathrm{a}}$ & $14.6^{\mathrm{a}}$ & $16.1^{\mathrm{a}}$ & 0.9 \\
$\mathrm{No}_{3}(\mathrm{mg} / \mathrm{l})$ & $0.1^{\mathrm{a}}$ & $0.1^{\mathrm{a}}$ & $0.1^{\mathrm{a}}$ & $0.1^{\mathrm{a}}$ & 0 \\
$\mathrm{Po}_{4}(\mathrm{mg} / \mathrm{l})$ & $0.1^{\mathrm{a}}$ & $0.1^{\mathrm{a}}$ & $0.1^{\mathrm{a}}$ & $0.1^{\mathrm{a}}$ & 0 \\
$\mathrm{NH}_{3}(\mathrm{mg} / \mathrm{l})$ & $0.9^{\mathrm{a}}$ & $0.9^{\mathrm{a}}$ & $0.1^{\mathrm{a}}$ & $0.1^{\mathrm{a}}$ & 0.1 \\
$\mathrm{Trans} .(\mathrm{cm})$ & $21.0^{\mathrm{ab}}$ & $23.1^{\mathrm{a}}$ & $22.3^{\mathrm{a}}$ & $19.1^{\mathrm{b}}$ & 1.6 \\
\hline
\end{tabular}

Means followed by different letters along the rows differ significantly at $\mathrm{P}>0.05$ by Student Newman -keul Test 
Table 3: Monthly Variation of Physico-chemical Parameters of CAAS Dam

\begin{tabular}{|c|c|c|c|c|c|c|c|c|c|c|c|c|c|}
\hline Months/Parameters & Jan & Feb & Mar & Apr & May & Jun & Jul & Aug & Sep & Oct & Nov & Dec & $\pm \mathrm{SE}$ \\
\hline $\operatorname{AT}\left({ }^{0} \mathrm{C}\right)$ & $26.0^{\mathrm{d}}$ & $34.5^{\mathrm{bc}}$ & $34.5^{b c}$ & $34.3^{\mathrm{c}}$ & $34.8^{\mathrm{bc}}$ & $25.5^{\mathrm{d}}$ & $31.5^{\mathrm{c}}$ & $31.5^{\mathrm{c}}$ & $38^{\mathrm{ab}}$ & $38^{\mathrm{ab}}$ & $40.3^{\mathrm{a}}$ & $34.5^{\mathrm{bc}}$ & 0.8 \\
\hline WT $\left({ }^{0} \mathrm{C}\right)$ & $22.3^{\mathrm{d}}$ & $25.8^{\mathrm{c}}$ & $25.8^{\mathrm{c}}$ & $30.8^{\mathrm{ab}}$ & $32.3^{\mathrm{ab}}$ & $30.0^{\mathrm{b}}$ & $32.0^{\mathrm{ab}}$ & $27.3^{\mathrm{c}}$ & $33.3^{\mathrm{a}}$ & $32.5^{\mathrm{ab}}$ & $32.0^{\mathrm{ab}}$ & $26.8^{\mathrm{c}}$ & 0.6 \\
\hline $\mathrm{pH}$ & $7.9^{\mathrm{a}}$ & $7.7^{\mathrm{ab}}$ & $6.5^{\mathrm{c}}$ & $6.8^{\mathrm{bc}}$ & $7.3^{\mathrm{abc}}$ & $6.4^{\mathrm{c}}$ & $7.6^{\mathrm{ab}}$ & $6.4^{c}$ & $7.2^{\mathrm{abc}}$ & $7.3^{\mathrm{abc}}$ & $7.1^{\mathrm{abc}}$ & $7.4^{\mathrm{abc}}$ & 0.2 \\
\hline Cond. $(\mu \mathrm{s} / \mathrm{cm})$ & $51.8^{\mathrm{ab}}$ & $56.5^{\mathrm{ab}}$ & $113.5^{\mathrm{ab}}$ & $130.5^{\mathrm{a}}$ & $120.8^{\mathrm{ab}}$ & $85.5^{\mathrm{ab}}$ & $39.3^{\mathrm{b}}$ & $58.0^{\mathrm{ab}}$ & $95.3^{\mathrm{ab}}$ & $96.8^{\mathrm{ab}}$ & $97.3^{\mathrm{ab}}$ & $59.5^{\mathrm{ab}}$ & 17.1 \\
\hline $\mathrm{DO}(\mathrm{mg} / \mathrm{l})$ & $9.5^{\mathrm{ab}}$ & $9.5^{\mathrm{ab}}$ & $8.5^{\mathrm{ab}}$ & $8.5^{\mathrm{ab}}$ & $9.0^{\mathrm{ab}}$ & $6.0^{\mathrm{b}}$ & $9.3^{\mathrm{ab}}$ & $9.3^{\mathrm{ab}}$ & $10.8^{\mathrm{ab}}$ & $10.8^{\mathrm{ab}}$ & $8.5^{\mathrm{ab}}$ & $12.0^{\mathrm{a}}$ & 0.9 \\
\hline $\mathrm{BOD}(\mathrm{mg} / \mathrm{l})$ & $6.3^{\mathrm{a}}$ & $6.0^{\mathrm{a}}$ & $4.5^{\mathrm{a}}$ & $6.3^{\mathrm{a}}$ & $6.0^{\mathrm{a}}$ & $3.4^{\mathrm{a}}$ & $4.5^{\mathrm{a}}$ & $3.3^{\mathrm{a}}$ & $5.5^{\mathrm{a}}$ & $6.0^{\mathrm{a}}$ & $5.0^{\mathrm{a}}$ & $6.3^{\mathrm{a}}$ & 0.6 \\
\hline $\mathrm{TH}(\mathrm{mg} / \mathrm{l})$ & $25.5^{\mathrm{c}}$ & $24.0^{\mathrm{c}}$ & $40.0^{\mathrm{bc}}$ & $26.0 \mathrm{c}$ & $24.5^{\mathrm{c}}$ & $52.0^{\mathrm{ab}}$ & $64.0^{\mathrm{a}}$ & $42.5 \mathrm{bc}$ & $39.5^{\mathrm{bc}}$ & $45.0^{\mathrm{bc}}$ & $34.5^{\mathrm{bc}}$ & $31.0^{\mathrm{bc}}$ & 5.2 \\
\hline ALK (mg/l) & $15.5^{\mathrm{ab}}$ & $12.5^{\mathrm{b}}$ & $20.5^{\mathrm{ab}}$ & $19.5^{\mathrm{ab}}$ & $17.5^{\mathrm{ab}}$ & $28.5^{\mathrm{a}}$ & $17.5^{\mathrm{ab}}$ & $16.5^{\mathrm{ab}}$ & $17.0^{\mathrm{ab}}$ & $24.5^{\mathrm{ab}}$ & $18.5^{\mathrm{ab}}$ & $17.0^{\mathrm{ab}}$ & 3.2 \\
\hline $\mathrm{Cl}^{-}(\mathrm{mg} / \mathrm{l})$ & $14.1^{\mathrm{b}}$ & $15.3^{\mathrm{ab}}$ & $15.5^{\mathrm{ab}}$ & $16.1^{\mathrm{ab}}$ & $15.1^{\mathrm{ab}}$ & $22.3^{\mathrm{a}}$ & $15.5^{\mathrm{ab}}$ & $8.3^{\mathrm{b}}$ & $14.6^{\mathrm{b}}$ & $14.9^{\mathrm{ab}}$ & $13.1^{\mathrm{b}}$ & $12.5^{\mathrm{b}}$ & 1.7 \\
\hline $\mathrm{No}_{3}(\mathrm{mg} / \mathrm{l})$ & $0.1^{\mathrm{a}}$ & $0.1^{\mathrm{a}}$ & $0.1^{\mathrm{a}}$ & $0.1^{\mathrm{a}}$ & $0.1^{\mathrm{a}}$ & $0.1^{\mathrm{a}}$ & $0.2^{\mathrm{a}}$ & $0.2^{\mathrm{a}}$ & $0.2^{\mathrm{a}}$ & $0.2^{\mathrm{a}}$ & $0.1^{\mathrm{a}}$ & $0.1^{\mathrm{a}}$ & 0 \\
\hline $\mathrm{Po}_{4}(\mathrm{mg} / \mathrm{l})$ & $0.1^{\mathrm{a}}$ & $0.1^{\mathrm{a}}$ & $0.1^{\mathrm{a}}$ & $0.1^{\mathrm{a}}$ & $0.2^{\mathrm{a}}$ & $0.1^{\mathrm{a}}$ & $0.1^{\mathrm{a}}$ & $0.1^{\mathrm{a}}$ & $0.1^{\mathrm{a}}$ & $0.1^{\mathrm{a}}$ & $0.1^{\mathrm{a}}$ & $0.1^{\mathrm{a}}$ & 0 \\
\hline $\mathrm{NH}_{3}(\mathrm{mg} / \mathrm{l})$ & $0.7^{\mathrm{cd}}$ & $0.9^{\mathrm{bcd}}$ & $1.1^{\mathrm{abcd}}$ & $1.1^{\mathrm{abcd}}$ & $1.2^{\mathrm{abc}}$ & $0.7^{\mathrm{cd}}$ & $1.3^{\mathrm{ab}}$ & $1.4^{\mathrm{ab}}$ & $1.3^{\mathrm{ab}}$ & $1.1^{\mathrm{abcd}}$ & $0.7^{\mathrm{d}}$ & $0.7^{\mathrm{d}}$ & 0.1 \\
\hline Trans. (cm) & $24.5^{\mathrm{ab}}$ & $23.8^{\mathrm{ab}}$ & $23.8^{\mathrm{ab}}$ & $18.0^{\mathrm{bcd}}$ & $22.5^{\mathrm{abc}}$ & $14.0^{\mathrm{d}}$ & $17.8^{\mathrm{bcd}}$ & $16.3^{\mathrm{cd}}$ & $23.5^{\mathrm{ab}}$ & $29.5^{\mathrm{a}}$ & $24.3^{\mathrm{ab}}$ & $18.8^{\mathrm{bcd}}$ & 1.6 \\
\hline
\end{tabular}

Means followed by different letters along the row differ significantly at $\mathrm{P}>0.05$ by Student - Newman - keul Test 
Table 4: Stations Variation of Physico-chemical Parameters for River Kaduna

\begin{tabular}{llllll}
\hline Stations/Parameters & 1 & 2 & 3 & 4 & \pm SE \\
\hline AT $(0 \mathrm{C})$ & $34.0 \mathrm{a}$ & $31.2 \mathrm{~b}$ & $33.2 \mathrm{ab}$ & $33.0 \mathrm{ab}$ & 0.6 \\
WT $(0 \mathrm{C})$ & $30.3 \mathrm{a}$ & $28.2 \mathrm{~b}$ & $29.5 \mathrm{ab}$ & $28.8 \mathrm{ab}$ & 0.5 \\
pH & $7.1 \mathrm{a}$ & $7.0 \mathrm{a}$ & $7.1 \mathrm{a}$ & $7.2 \mathrm{a}$ & 0.1 \\
Cond. $(\mu \mathrm{s} / \mathrm{cm})$ & $119.1 \mathrm{a}$ & $141.5 \mathrm{a}$ & $143.6 \mathrm{a}$ & $154.6 \mathrm{a}$ & 9.3 \\
DO $(\mathrm{mg} / \mathrm{l})$ & $9.2 \mathrm{a}$ & $9.7 \mathrm{a}$ & $9.1 \mathrm{a}$ & $10.0 \mathrm{a}$ & 0.7 \\
OD $(\mathrm{mg} / \mathrm{l})$ & $5.8 \mathrm{a}$ & $6.1 \mathrm{a}$ & $5.4 \mathrm{a}$ & $6.5 \mathrm{a}$ & 0.5 \\
TH $(\mathrm{mg} / \mathrm{l})$ & $59.2 \mathrm{a}$ & $54.5 \mathrm{a}$ & $56.9 \mathrm{a}$ & $61.5 \mathrm{a}$ & 1.9 \\
ALK $(\mathrm{mg} / \mathrm{l})$ & $26.2 \mathrm{~b}$ & $29.2 \mathrm{~b}$ & $28.8 \mathrm{~b}$ & $34.7 \mathrm{a}$ & 1.6 \\
Cl- $(\mathrm{mg} / \mathrm{l})$ & $16.9 \mathrm{a}$ & $15.5 \mathrm{a}$ & $15.6 \mathrm{a}$ & $15.6 \mathrm{a}$ & 0.7 \\
No3 $(\mathrm{mg} / \mathrm{l})$ & $0.2 \mathrm{a}$ & $0.1 \mathrm{a}$ & $0.1 \mathrm{a}$ & $0.1 \mathrm{a}$ & 0 \\
Po4 $(\mathrm{mg} / \mathrm{l})$ & $0.1 \mathrm{a}$ & $0.1 \mathrm{a}$ & $0.1 \mathrm{a}$ & $0.0 \mathrm{a}$ & 0 \\
NH3 $(\mathrm{mg} / \mathrm{l})$ & $0.2 \mathrm{a}$ & $0.2 \mathrm{a}$ & $0.2 \mathrm{a}$ & $0.2 \mathrm{a}$ & 0 \\
Trans. $(\mathrm{cm})$ & $21.5 \mathrm{a}$ & $22.6 \mathrm{a}$ & $22.3 \mathrm{a}$ & $22.9 \mathrm{a}$ & 0.8
\end{tabular}

Means followed by different letters along the rows differ significantly at $\mathrm{P}>0.05$ by Student - Newman - keul Test

Table 5: Monthly variation of Physico-chemical Parameters for River Kaduna

\begin{tabular}{|c|c|c|c|c|c|c|c|c|c|c|c|c|c|}
\hline Months/Parameters & Jan & Feb & Mar & Apr & May & Jun & Jul & Aug & Sep & Oct & Nov & Dec & $\pm \mathrm{SE}$ \\
\hline $\operatorname{AT}\left({ }^{0} \mathrm{C}\right)$ & $28.01^{\mathrm{cf}}$ & $32.0^{\mathrm{bcdc}}$ & $30.8^{\mathrm{cdcf}}$ & $34.3^{\mathrm{abcd}}$ & $35.6^{\mathrm{ab}}$ & $27.0^{\mathrm{f}}$ & $30.0^{\mathrm{dct}}$ & $30.0^{\mathrm{dcf}}$ & $34.8^{\mathrm{abc}}$ & $35.0^{\mathrm{abc}}$ & $38.8^{\mathrm{a}}$ & $37.8^{\mathrm{a}}$ & 1.1 \\
\hline $\mathrm{WT}\left({ }^{0} \mathrm{C}\right)$ & $22.5^{\mathrm{c}}$ & $26.5^{\mathrm{b}}$ & $25.3^{\mathrm{b}}$ & $30.8^{\mathrm{a}}$ & $33.3^{\mathrm{a}}$ & $30.5^{\mathrm{a}}$ & $31.0^{\mathrm{a}}$ & $27.5^{\mathrm{b}}$ & $31.0^{\mathrm{a}}$ & $30.8^{\mathrm{a}}$ & $31.5^{\mathrm{a}}$ & $30.0^{\mathrm{a}}$ & 0.8 \\
\hline $\mathrm{pH}$ & $7.5^{\mathrm{b}}$ & $7.9^{\mathrm{a}}$ & $6.6^{\mathrm{de}}$ & $6.9^{\mathrm{cd}}$ & $7.0^{\mathrm{cd}}$ & $6.6^{\mathrm{de}}$ & $7.2^{\mathrm{bc}}$ & $7.2^{\mathrm{bc}}$ & $7.2^{\mathrm{bc}}$ & $7.2^{\mathrm{bc}}$ & $6.6^{\mathrm{de}}$ & $6.5^{\mathrm{e}}$ & 0.1 \\
\hline Cond. $(\mu \mathrm{s} / \mathrm{cm})$ & $163.0^{\mathrm{bcd}}$ & $174.5^{\mathrm{bc}}$ & $314.5^{\mathrm{a}}$ & $331.3^{\mathrm{a}}$ & $192.3^{\mathrm{b}}$ & $40.5^{\mathrm{g}}$ & $40.3^{\mathrm{g}}$ & $38.3^{\mathrm{g}}$ & $65.3^{\mathrm{gf}}$ & $76.3^{\mathrm{cfg}}$ & $111.3^{\mathrm{dcf}}$ & $111.3^{\mathrm{dcf}}$ & 16.1 \\
\hline $\mathrm{DO}(\mathrm{mg} / \mathrm{l})$ & $9.5^{\mathrm{ab}}$ & $8.3^{\mathrm{ab}}$ & $7.5^{\mathrm{ab}}$ & $10.5^{\mathrm{ab}}$ & $9.0^{\mathrm{ab}}$ & $6.0^{\mathrm{b}}$ & $9.0^{\mathrm{ab}}$ & $9.3^{\mathrm{ab}}$ & $9.8^{\mathrm{ab}}$ & $11.0^{\mathrm{ab}}$ & $11.5^{\mathrm{ab}}$ & $12.5^{\mathrm{a}}$ & 1.1 \\
\hline $\mathrm{BOD}(\mathrm{mg} / \mathrm{l})$ & $6.0^{\mathrm{a}}$ & $5.3^{\mathrm{a}}$ & $5.5^{\mathrm{a}}$ & $7.8^{\mathrm{a}}$ & $6.0^{\mathrm{a}}$ & $3.9^{\mathrm{a}}$ & $4.3^{\mathrm{a}}$ & $5.5^{\mathrm{a}}$ & $5.8^{\mathrm{a}}$ & $7.3^{\mathrm{a}}$ & $7.5^{\mathrm{a}}$ & $6.8^{\mathrm{a}}$ & 0.8 \\
\hline $\mathrm{TH}(\mathrm{mg} / \mathrm{l})$ & $54.0^{\mathrm{cd}}$ & $55.5^{\mathrm{cd}}$ & $74.5^{\mathrm{ab}}$ & $65.0^{\mathrm{bc}}$ & $51.0^{\text {cde }}$ & $82.0^{\mathrm{a}}$ & $69.0^{\mathrm{b}}$ & $54.5^{\mathrm{cd}}$ & $51.5^{\text {cde }}$ & $55.8^{\mathrm{cd}}$ & $43.5^{\mathrm{de}}$ & $40.0^{\mathrm{e}}$ & 3.3 \\
\hline ALK (mg/l) & $32.5^{\mathrm{bc}}$ & $35.0^{\mathrm{bc}}$ & $59.0^{\mathrm{a}}$ & $34.5^{\mathrm{bc}}$ & $39.5^{\mathrm{b}}$ & $15.5^{\mathrm{d}}$ & $15.5^{\mathrm{d}}$ & $16.0^{\mathrm{d}}$ & $25.5^{\mathrm{cd}}$ & $35.5^{\mathrm{bc}}$ & $26.0^{\mathrm{cd}}$ & $22.0^{\mathrm{d}}$ & 2.7 \\
\hline $\mathrm{Cl}^{-}(\mathrm{mg} / \mathrm{l})$ & $13.9^{\mathrm{b}}$ & $15.8^{\mathrm{b}}$ & $15.8^{\mathrm{b}}$ & $16.4^{\mathrm{b}}$ & $16.3^{\mathrm{b}}$ & $27.4^{\mathrm{a}}$ & $25.7^{\mathrm{a}}$ & $6.9^{c}$ & $12.9^{\mathrm{b}}$ & $13.7^{\mathrm{b}}$ & $13.7^{\mathrm{b}}$ & $12.9^{\mathrm{b}}$ & 1.1 \\
\hline $\mathrm{No}_{3}(\mathrm{mg} / \mathrm{l})$ & $0.1^{\mathrm{a}}$ & $0.2^{\mathrm{a}}$ & $0.1^{\mathrm{a}}$ & $0.1^{\mathrm{a}}$ & $0.1^{\mathrm{a}}$ & $0.2^{\mathrm{a}}$ & $0.1^{\mathrm{a}}$ & $0.1^{\mathrm{a}}$ & $0.1^{\mathrm{a}}$ & $0.4^{\mathrm{a}}$ & $0.1^{\mathrm{a}}$ & $0.1^{\mathrm{a}}$ & 0.1 \\
\hline $\mathrm{Po}_{4}(\mathrm{mg} / \mathrm{l})$ & $0.1^{\mathrm{a}}$ & $0.1^{\mathrm{a}}$ & $0.0^{\mathrm{a}}$ & $0.0^{\mathrm{a}}$ & $0.1^{\mathrm{a}}$ & $0.0^{\mathrm{a}}$ & $0.0^{\mathrm{a}}$ & $0.0^{\mathrm{a}}$ & $0.1^{\mathrm{a}}$ & $0.0^{\mathrm{a}}$ & $0.1^{\mathrm{a}}$ & $0.1^{\mathrm{a}}$ & 0 \\
\hline $\mathrm{NH}_{3}(\mathrm{mg} / \mathrm{l})$ & $0.2^{\mathrm{b}}$ & $0.2^{\mathrm{b}}$ & $0.2^{\mathrm{b}}$ & $0.2^{\mathrm{b}}$ & $0.4^{\mathrm{a}}$ & $0.1^{\mathrm{b}}$ & $0.2^{\mathrm{b}}$ & $0.3^{\mathrm{ab}}$ & $0.1^{\mathrm{b}}$ & $0.2^{\mathrm{b}}$ & $0.2^{\mathrm{b}}$ & $0.2^{\mathrm{b}}$ & 0 \\
\hline Trans. $(\mathrm{cm})$ & $29.5^{\mathrm{a}}$ & $28.3^{\mathrm{a}}$ & $28.3^{\mathrm{a}}$ & $27.8^{\mathrm{a}}$ & $24.3^{\mathrm{ab}}$ & $14.0^{\mathrm{c}}$ & $14.3^{\mathrm{c}}$ & $18.5^{\mathrm{bc}}$ & $18.5^{\mathrm{bc}}$ & $24.5^{\mathrm{ab}}$ & $21.0^{\mathrm{b}}$ & $19.0^{\mathrm{bc}}$ & 1.5 \\
\hline
\end{tabular}

Means followed by different letters along the row differ significantly at $\mathrm{P}>0.05$ by Student - Newman - keul Test 
Assessment of Physico-chemical Parameters of River Kaduna.................JUTIKU et al.

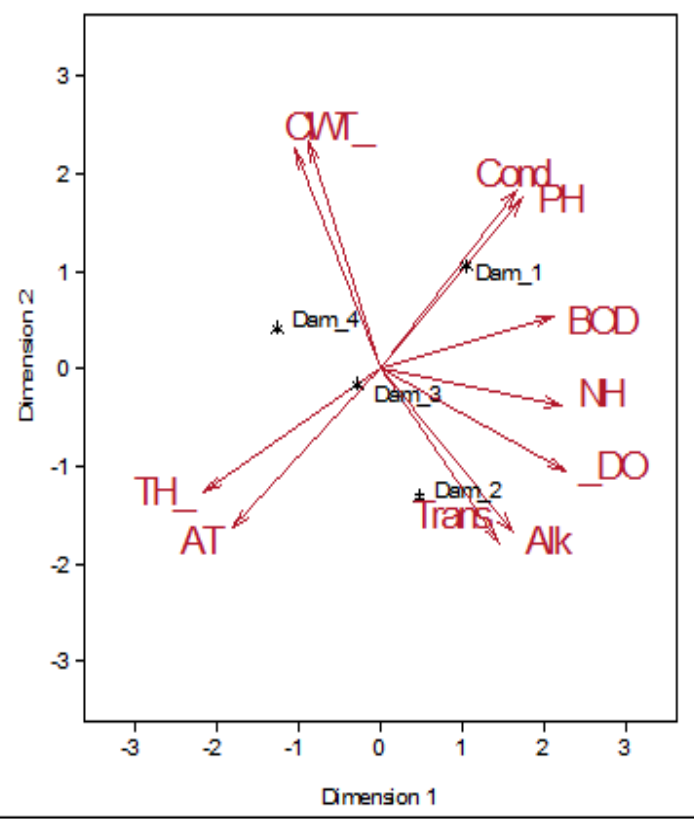

Figure 2: Graph showing Correlation of Physico-chemical parameters of CAAS Dam

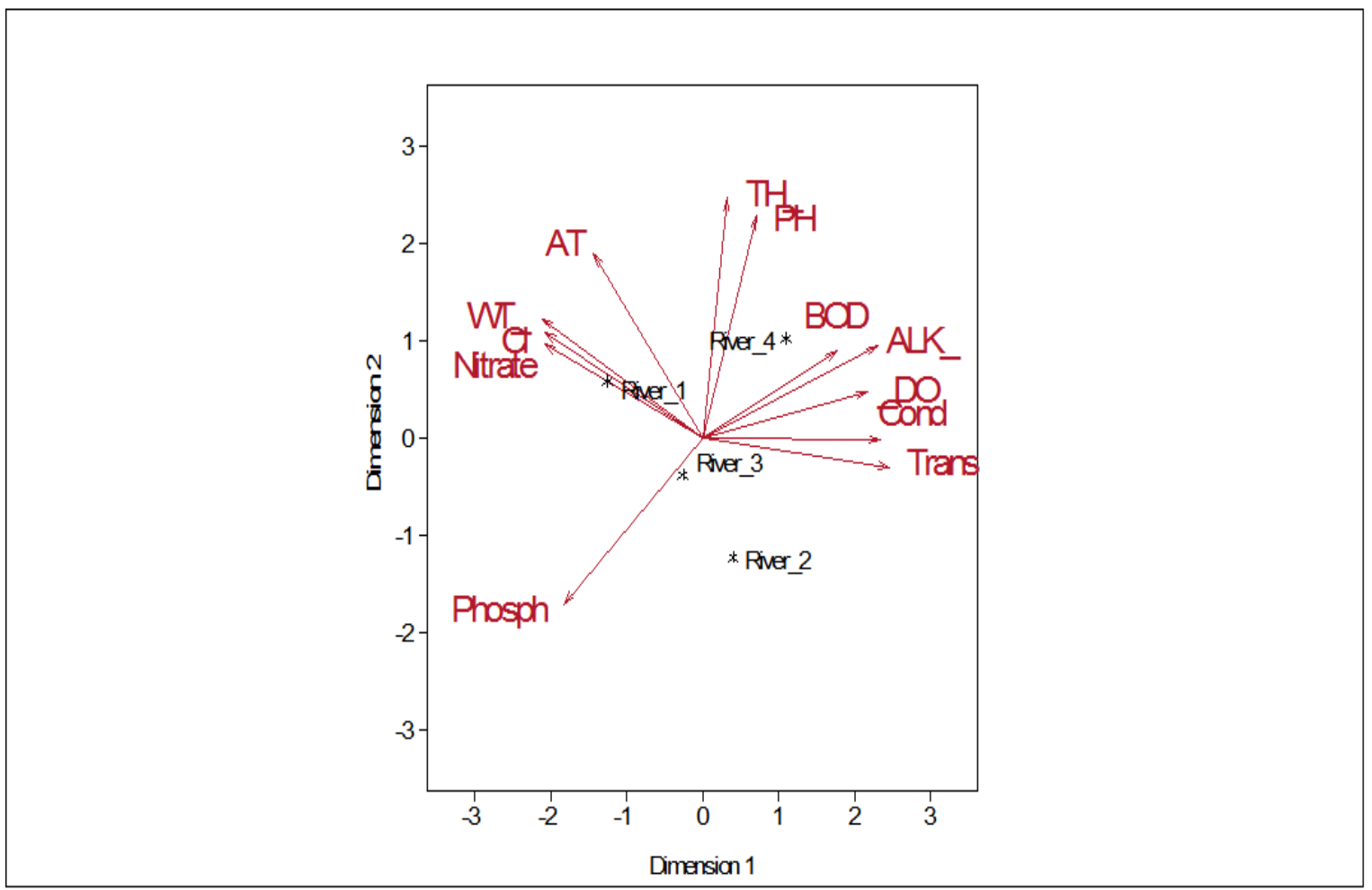

Figure 3: Graph showing Correlation of Physico-chemical parameters of River Kaduna 


\section{Conclusion}

Physico-chemical parameters in the CAAS Dam and River Kaduna result revealed that some parameters were above the recommended acceptable limit, it is therefore recommended that urgent management steps be taken to arrest future deleterious effects and sustainability of the water body for fish culture.

\section{References}

Asonye, C.C., Okonlie, N. P., Okenwa, E. E. and Iwuanyanwu, U. G. (2007). Some physico-chemical characteristics and heavy metal profiles of Nigeria rivers, streams and waterways. African journal of Biotechnology, 6(5): 617-624.

Edegbene, A.O., Arimoro, F.O., Nwaka, K.H., Omovah, G.O., Ogidiaka. E. and Abolagba, O.J. (2012). The physical and chemicals characteristic of Atapko River, Niger Delta, Nigeria. Journal of Aquatic sciences, 27(2): 159 - 172.

Edegbene, A.O and Arimoro, F.O. (2012). Ecological Status of Owan River, Southern Nigeria Using Aquatic Insects as Bioindicators. Journal of Aquatic Sciences, 27(2): $99-111$.

Edokpayi, C.A. and Osimen, E.C. (2002). The impact of impoundment on the physical and chemical hydrology of Ibiekuma steam in southern Nigeria. Tropical Ecology, 43(2): 287-296.

Kataria, H.C. (2004). Analytical Study of Trace Elements in Groundwater of
Bhopal, I.J. Env. Prot. 24(12): 894896

Mahre, M.Y., Akan, J.C., Moses, E.A. and Ogugbuaja, V.O. (2007). Pollution indicators in River Kaduna, Kaduna state, Nigeria. Trend in Applied Sciences Research, 2(4): 304-311.

Ojutiku, R.O. and Kolo, R.J. (2011). Temporal and spatial variations in some physic-chemical parameters of River chanchaga, Niger State, Nigeria. Journal of Applied Bioscience, 47: 3242 - 3255.

Ovie, S.I. (2011). A synopsis of the zooplankton fauna of Lakes Kainji and Jebba. In: Forty years on Lake Kainji fisheries research. Eds: Raji, A. Okaeme, A. N. and Ibeun, M. O. NIFFR, New-Bussa, Nigeria. Vol. 1: 133-143.

Rajagopal, T., Thangamani, A., Sevarkodiyone, S., Sekar, M. and Archunan, G. (2011). Zooplankton diversity and physico-chemical conditions in three perennial ponds of Virudhunagar district, Tamilnadu. Journal of Environmental Biology, 31: 265272.

Trivedy, R.K., Khatawkar, S.D., Kulkarni, A.Y and Shastri, A.C. (1990). River pollution in India, Ashish Publishing House, New Delhi, 26, 99.

Wade, J.W. (1992). The relationship between temperature, food intake and growth of brown trout Salmon trutta (L) fed natural and artificial pelleted diet in earth ponds. Journal of aquatic sciences, 14(1): 29 - 33. 